\title{
A new method for operations of interval numbers
}

\author{
Zhigeng Fang \\ School of Economics and Management \\ Nanjing University of Aeronautics and Astronautics \\ Nanjing, China \\ zhigengfang@163.com
}

\author{
Yingjie Yang \\ Centre for Computational Intelligence \\ De Montfort University \\ Leicester, England \\ yyang@dmu.ac.uk
}

\begin{abstract}
The present interval arithmetic often results in unnecessary diameter expansions in interval operations. To solve this problem, this paper defines a new operation of intervals using their algebraic representations. On the basis of algebraic interval representations, the arithmetic operations are investigated and formulated. Then, the capability of the proposed operation methods in solving unnecessary diameter expansions is analyzed. An application of the proposed model to a typical game problem is provided in the end to demonstrate the feasibility of the proposed model.
\end{abstract}

Keywords- Interval numbers; Standard interval numbers; Arithmetic operations

\section{INTRODUCTION (HEADING 1)}

Since Young ${ }^{[16]}$ proposed the concept of interval numbers, it has attracted the attention of a large number of scholars. The arithmetic operations and sorting of interval numbers have been the focus of many related research works. However, most of the available operation models of interval numbers suffer from high complexity, low efficiency and poor accuracy, and etc. In the same time, there is no universally accepted way on sorting interval numbers yet.

Moore ${ }^{[10-11]}$ and many other scholars have studied interval numbers in depth, and made significant contribution in this field. Zhang ${ }^{[18]}$ proposed a method to convert interval arithmetic operations into matrix operations. Redrycz and Park ${ }^{[13]}$ applied interval arithmetic and fuzzy sets to deal with classification. Considering the special features of timed intervals, Chen and Dong ${ }^{[2]}$ also developed a system based on a generic theorem prover, Prototype Verification System, to assist formal verification of timed interval calculus at a high grade of automation. Deng Julong ${ }^{[3]}$ illustrated the differences and similarity between interval numbers and interval grey numbers. The application of interval numbers has been extended to many fields. For instance, non-linear computation based on interval numbers, computational intelligence, classification, pattern identification and spatial reasoning based on interval numbers, etc.

The relationship between two interval numbers includes three different situations: non-intersection, intersection, and inclusion. Many models have been proposed to sort interval numbers in [1], [7], [9], [10], [12], [14], [17], [18], [19].

Japanese scholar Nakahara et al. ${ }^{[12]}$ defined a possibility measurement for comparing two interval numbers, and applied it in solving a fuzzy mathematical programming problem. Utilizing min-transitive fuzzy leftness relations, Kundu ${ }^{[9]}$ derived a partial order between two interval numbers. This method may give unreasonable results and low resolutions for comparisons between some special interval numbers. Based on mean value and interval diameter, Sengupta and $\mathrm{Pal}^{[14]}$ proposed an acceptability index, but it does not work well in comparing multiple interval numbers. Zeshui $\mathrm{Xu}$ et al. ${ }^{[15]}$ suggested a possibility measurement for comparing two interval numbers, and derived a possibility matrix to sort interval numbers. Based on Xu's work, Jijun Zhang ${ }^{[17]}$ proposed a method of sorting interval numbers using relative superiority and improved the resolution. Obviously, most available methods show some limitations, and it is worthy of further study on interval numbers.

Great achievements have been obtained in interval related research. It has been widely used in decision-making, management, engineering and other fields. However, there are still serious problems in the available models for representation and operations of interval numbers. While, when conducting interval operations, it is easy to amplify the uncertain degree so that the usability of the result is poor. Here we investigate a different presentation and associated arithmetic operations of interval numbers in order to overcome the problems.

\section{ANALYSIS OF PROBLEMS IN TRADITIONAL INTERVAL ARITHMETIC OPERATIONS}

Definition 1 Let $F\left(\Theta_{k_{i}}\right)=\Theta_{k_{i}}\left\{A_{1}, A_{2}, \cdots, A_{n}\right\}$ $=\left[A_{L}^{\left(K_{i}\right)}, A_{R}^{\left(K_{i}\right)}\right]$ be an arithmetic operation of interval numbers $A_{s}=\left[a_{s}, b_{s}\right], s=1,2, \cdots \quad F\left(\Theta_{k_{i}}\right)$ and $F\left(\Theta_{k_{j}}\right)$ refer to different models of the same arithmetic operation. $H_{k_{i}}=\left|A_{R}^{K_{i}}-A_{L}^{K_{i}}\right|$ and $H_{k_{j}}=\left|A_{R}^{K_{j}}-A_{L}^{K_{j}}\right|$ denote the diameters of model $F\left(\Theta_{k_{i}}\right)$ and $F\left(\Theta_{k_{j}}\right)$. If $H_{k_{i}} \geq H_{k_{j}}$ and $F\left(\Theta_{k_{j}}\right)$ does not exclude any possible resulting values, then we call model $F\left(\Theta_{k_{i}}\right)$ causes amplification distortion compared with model $F\left(\Theta_{k_{j}}\right)$. 
Proposition 1 The classical interval arithmetic operations may cause amplification distortion.

This proposition can be proved by an example.

Example 1. Give interval numbers $A_{1}=[1,5], A_{2}=[2,5], \quad A_{3}=1, \quad A_{4}=2$, and we want to get $\mathrm{x}: x=\frac{A_{1} \cdot A_{2}+A_{1} \cdot A_{2} \cdot A_{3}}{A_{2} \cdot A_{4}+A_{1} \cdot A_{2} \cdot A_{3}}$.

Solution: (1) Using the classic interval arithmetic $k_{1}$, we have

$$
x=\frac{A_{1} \cdot A_{2}+A_{1} \cdot A_{2} \cdot A_{3}}{A_{2} \cdot A_{4}+A_{1} \cdot A_{2} \cdot A_{3}}=\frac{[1,5] \cdot[2,5]+[1,5] \cdot[2,5] \cdot 1}{[2,5] \cdot 2+[1,5] \cdot[2,5] \cdot 1}=\left[\frac{4}{35}, \frac{50}{6}\right]
$$

(2) In mathematical optimization algorithm $k_{2}$,

let $x=f\left(A_{i}\right), \quad i=1,2,3 \quad$, then $x^{\prime}=\left[\min \left(f\left(A_{i}\right)\right), \max \left(f\left(A_{i}\right)\right)\right], \quad i=1,2,3$.

$$
\begin{aligned}
\min \left(f\left(A_{i}\right)\right. & =\min \left(\frac{[1,5] \cdot[2,5]+[1,5] \cdot[2,5] \cdot 1}{[2,5] \cdot 2+[1,5] \cdot[2,5] \cdot 1}\right) \\
& =\min \left(\frac{[1,5]+[1,5]}{2+[1,5]}\right)=\frac{2}{3} \\
\max \left(f\left(A_{i}\right)\right) & =\max \left(\frac{[1,5] \cdot[2,5]+[1,5] \cdot[2,5] \cdot 1}{[2,5] \cdot 2+[1,5] \cdot[2,5] \cdot 1}\right) \\
& =\max \left(\frac{[1,5]+[1,5]}{2+[1,5]}\right)=\frac{10}{7}
\end{aligned}
$$

Sum up Equation 1.2 and Equation 1.3, we can conclude that

$$
x^{\prime}=\left[\min \left(f\left(A_{i}\right)\right), \max \left(f\left(A_{i}\right)\right)\right]=\left[\frac{2}{3}, \frac{10}{7}\right]
$$

Comparing Equation 1.1 with Equation 1.4, we know that the diameter of $x$ in Equation 1.1 is larger than that of $x^{\prime}$ in Equation 1.4,

$$
\frac{4}{35}<\left[\frac{2}{3}, \frac{10}{7}\right]<\frac{50}{6}
$$

Thus, compared with the mathematical optimization algorithms $k_{2}$, the classic interval arithmetic $k_{1}$ causes the amplification distortion of the results. The mathematical optimization algorithm is not relied on the interval arithmetic, so its result is more reliable.

Proposition 2 The amplification distortion of interval arithmetic operations is caused by the inconsistent values of the same interval if it appears more than once in the same arithmetic operation.

This theorem can be proved by example.

Example2. In equation 1.1 , if $A_{1}$ and $A_{2}$ take their maximum values in the numerator and their minimum values in the denominator, we can get the maximum of $x$, $\max \{x\}$ as shown in Equation 1.5.1. Similarly, we can get the minimum of $x, \min \{x\}$ as shown in Equation 1.5.2.

$$
\begin{aligned}
& \max \{x\}=\max \left\{\frac{A_{1} \cdot A_{2}+A_{1} \cdot A_{2} \cdot A_{3}}{A_{2} \cdot A_{4}+A_{1} \cdot A_{2} \cdot A_{3}}\right\}=\frac{50}{6} \\
& \min \{x\}=\min \left\{\frac{A_{1} \cdot A_{2}+A_{1} \cdot A_{2} \cdot A_{3}}{A_{2} \cdot A_{4}+A_{1} \cdot A_{2} \cdot A_{3}}\right\}=\frac{4}{35}
\end{aligned}
$$

Comparing with Equation 1.1, Equation 1.5 gives the same result as equation 1.1. It is the different values taken by the same interval in numerator and denominator that produces the amplification of the diameter in the result.

If we take the identical value for the same interval number in numerator and denominator, then the result is different from Equation 1.5. Take $A_{2}$ as identical in both the numerator and denominator, we get Equation 1.6.

$x=\frac{A_{1} \cdot A_{2}+A_{1} \cdot A_{2} \cdot A_{3}}{A_{2} \cdot A_{4}+A_{1} \cdot A_{2} \cdot A_{3}}=\frac{[1,5]+[1,5]}{2+[1,5]}$

Furthermore, if we consider all $A_{1}$ in Equation 1.6 as identical number, and evaluate the left endpoint value (minimum) and the right endpoint value (maximum ) of $\mathrm{x}$, we get Equation 1.7。

$$
\begin{aligned}
& \min \{x\}=\min \left\{\frac{A_{1}+A_{1} \cdot A_{3}}{A_{4}+A_{1} \cdot A_{3}}\right\}=\frac{2}{3} \\
& \max \{x\}=\max \left\{\frac{A_{1}+A_{1} \cdot A_{3}}{A_{4}+A_{1} \cdot A_{3}}\right\}=\frac{10}{7}
\end{aligned}
$$

From Equation 1.7, we have

$x=[\min \{x\}, \max \{x\}]=\left[\frac{2}{3}, \frac{10}{7}\right]$

Comparing Equation 1.8 with Equation 1.4, we know that this result is the same as the mathematical optimization algorithm $k_{2}$. Therefore, it is the multiple values of the same interval that cause the amplification distortion of the result in the traditional interval arithmetic operations. 


\section{ALGEBRAIC REPRESENTATION OF INTERVAL NUMBERS}

Considering the aforementioned problems in interval arithmetic operations, it is necessary to find a way to avoid the amplification distortion in interval arithmetic operations. To do this, we need a new way to represent interval numbers during their operations to avoid the false amplification of resulting diameters.

Definition 2.1 Given an interval number $A_{i}=\left[a_{i}, b_{i}\right], \quad i=1,2, \cdots$, we can represent it as Equation 2.1. Here, $a_{i}$ is called the white part (which means information is completely clear) of $A_{i}, c_{i} \cdot \gamma_{i}$ is called grey part (which means information is not completely clear) of $A_{i}$, and $c_{i}$ is called grey coefficient, $\gamma_{i}$ is an unit interval $[0,1]$ specific to $A_{i}$

$$
A_{i}=a_{i}+c_{i} \cdot \gamma_{i}, \quad i=1,2, \cdots
$$

The representation of an interval number in the form of Equation 2.1 is called an algebraic interval number.

Theorem 2.1 For any given interval number $A_{i}=\left[a_{i}, b_{i}\right], a_{i} \leq b_{i}, i=1,2, \cdots$, there is an equivalent algebraic interval number in the form of definition 2.1 (see Equation 2.1).

Proof: Without loss of generality, we can transform (the process is called algebraic transformation) any interval number $A_{i}=\left[a_{i}, b_{i}\right], a_{i} \leq b_{i}, i=1,2, \cdots$ to its algebraic form as shown in Equation 2.2.

$$
\begin{aligned}
A_{i} & =\left[a_{i}, b_{i}\right]=\left[a_{i}, b_{i}\right]+a_{i}-a_{i} \\
& =a_{i}+\left[0, b_{i}-a_{i}\right]=a_{i}+\left(b_{i}-a_{i}\right) \cdot[0,1]
\end{aligned}
$$

If $c_{i}=b_{i}-a_{i}$ and $\gamma_{i} \in[0,1]$, then equation 2.2 can be written as the algebraic expression of interval numbers as shown in Equation 2.3,

$$
A_{i}=a_{i}+\left(b_{i}-a_{i}\right) \cdot \gamma_{i}=a_{i}+c_{i} \cdot \gamma_{i}
$$

Definition 2.4 Take a certain value for the unit interval number $\gamma_{i} \in[0,1]$ in an algebraic interval number $A_{i}=a_{i}+c_{i} \cdot \gamma_{i}$, value of $A_{i}$ can then be uniquely determined. We call this process as the value determination for $A_{i}=a_{i}+c_{i} \cdot \gamma_{i}$. If the values of the unit interval numbers are the same for different algebraic interval numbers or different situations of the same algebraic interval number, we call them consistent to each other in their value determination.

\section{OPERATIONS BETWEEN INTERVAL NUMBERS}

The representation of interval numbers in the form of algebraic interval numbers provides a convenient condition for its arithmetic operations. Based on the arithmetic operations of ordinary numbers, this section discusses the operations of algebraic interval numbers.

Definition 3.1 Let $F(\Theta)=\Theta\left\{A_{1}, A_{2}, \cdots, A_{n}\right\}$ be the operation result of some algebraic interval numbers $A_{i}=a_{i}+c_{i} \cdot \gamma_{i}, i=1,2, \ldots$ following traditional mathematics

for numbers, and $\gamma_{i}, \gamma_{i} \in[0,1], i=1,2, \cdots$ be their kernel values, the minimum and maximum values are shown as Equation 3.1

$$
\begin{aligned}
& \min \{F(\Theta)\}=\min \Theta\left\{A_{1}, \cdots, A_{n}\right\} \mid \gamma_{i}^{(0)}=c_{i}, c_{i} \in[0,1], i=1, \cdots, n \\
& \max \{F(\Theta)\}=\max \Theta\left\{A_{1}, \cdots, A_{n}\right\} \mid \begin{array}{l}
\gamma_{j}^{(0)}=c_{j}, c_{j} \in[0,1], j=1, \cdots, n
\end{array}
\end{aligned}
$$

Then the result of this operation is an interval number $A=[\min \{F(\Theta)\}, \max \{F(\Theta)\}]$.

Obviously, the dependency between kernel values of interval numbers can be easily identified in Equation (3.1), and hence the amplification of resulting diameters can be reduced. The result of Equation (3.1) is identical to the traditional interval number arithmetic if the kernel values of the involved interval numbers are independent to each other.

Theorem 3.1 Given interval numbers $A_{i}=\left[a_{i}, b_{i}\right],\left(a_{i} \leq b_{i}, i=1,2, \cdots\right) \quad, \quad A_{j}=\left[a_{j}, b_{j}\right] \quad$, $\left(a_{j} \leq b_{j}, j=1,2, \cdots\right) \quad$; the sum of $A_{i}$ and $A_{j}$ is 
$A_{i}+A_{j}=\left[\left(a_{i}+a_{j}\right),\left(b_{i}+b_{j}\right)\right]$ when algebraic interval numbers are employed as immediate representation in the operation.

Proof: transform $A_{i}=\left[a_{i}, b_{i}\right],\left(a_{i} \leq b_{i}, i=1,2, \cdots\right)$ and $A_{j}=\left[a_{j}, b_{j}\right], \quad\left(a_{j} \leq b_{j}, j=1,2, \cdots\right)$ and obtain their algebraic interval numbers, which are expressed as Equation 3.2, then sum up Equation 3.2.1 and Equation 3.2.2, the result is shown in Equation 3.3.

$$
\begin{gathered}
A_{i}=a_{i}+\left(b_{i}-a_{i}\right) \cdot \gamma_{i},\left(\gamma_{i} \in[0,1]\right) \quad(3.2 .1) \\
A_{j}=a_{j}+\left(b_{j}-a_{j}\right) \cdot \gamma_{j},\left(\gamma_{j} \in[0,1]\right)(3.2 .2) \\
A_{i}+A_{j}=\left(a_{i}+a_{j}\right)+\left[\left(b_{i}-a_{i}\right) \cdot \gamma_{i}+\left(b_{j}-a_{j}\right) \cdot \gamma_{j}\right] \\
\min \left(A_{i}+A_{j}\right)=\left.\left\{\left(a_{i}+a_{j}\right)+\left[\left(b_{i}-a_{i}\right) \cdot \gamma_{i}+\left(b_{j}-a_{j}\right) \cdot \gamma_{j}\right]\right\}\right|_{\gamma_{i}^{(0)}=0, \gamma_{j}^{(0)}=0} \\
=\left(a_{i}+a_{j}\right) \\
\max \left(A_{i}+A_{j}\right)=\left.\left\{\left(a_{i}+a_{j}\right)+\left[\left(b_{i}-a_{i}\right) \cdot \gamma_{i}+\left(b_{j}-a_{j}\right) \cdot \gamma_{j}\right]\right\}\right|_{\gamma_{i}^{(0)}=1, \gamma_{j}^{(0)}=1} \\
=\left(b_{i}+b_{j}\right) \quad
\end{gathered}
$$

Here, max or $\left.\min (\cdot)\right|_{\gamma_{i}^{(0)}=c_{i}, \gamma_{j}^{(0)}=c_{j}}$ means, when the values of $\gamma_{i}, \gamma_{j}$ are $c_{i}, c_{j}$ respectively, max or $\min (\cdot)$ is maximum or minimum.

Considering both equation 3.3.1 and equation 3.3.2, we Have $A_{i}+A_{j}=\left[\left(a_{i}+a_{j}\right),\left(b_{i}+b_{j}\right)\right]$.

Theorem 3.2 Given interval numbers $A_{i}=\left[a_{i}, b_{i}\right],\left(a_{i} \leq b_{i}, i=1,2, \cdots\right) \quad, \quad A_{j}=\left[a_{j}, b_{j}\right] \quad$, $\left(a_{j} \leq b_{j}, j=1,2, \cdots\right)$, then the difference of $A_{i}$ and $A_{j}$ is $A_{i}-A_{j}=\left[\left(a_{i}-b_{j}\right),\left(b_{i}-a_{j}\right)\right]$ when algebraic interval numbers are employed as immediate representation in the operation.

Theorem 3.3 Given interval numbers $A_{i}=\left[a_{i}, b_{i}\right],\left(a_{i} \leq b_{i}, i=1,2, \cdots\right) \quad, \quad A_{j}=\left[a_{j}, b_{j}\right] \quad$, $\left(a_{j} \leq b_{j}, j=1,2, \cdots\right)$, then the product of $A_{i}$ and $A_{j}$ is

$$
\begin{aligned}
A_{i} \cdot A_{j} & =\left[\min \left(A_{i} \cdot A_{j}\right), \max \left(A_{i} \cdot A_{j}\right)\right] \\
& =\left[\begin{array}{c}
\min \left\{\left(a_{i} \cdot a_{j}\right),\left(a_{i} \cdot b_{j}\right),\left(b_{i} \cdot b_{j}\right),\left(b_{i} \cdot a_{j}\right)\right\} \\
, \max \left\{\left(a_{i} \cdot a_{j}\right),\left(a_{i} \cdot b_{j}\right),\left(b_{i} \cdot b_{j}\right),\left(b_{i} \cdot a_{j}\right)\right\}
\end{array}\right]
\end{aligned}
$$

when algebraic interval numbers are employed as intermediate representations in the operation.

Proof: transform $A_{i}=\left[a_{i}, b_{i}\right],\left(a_{i} \leq b_{i}, i=1,2, \cdots\right) \quad$ and $A_{j}=\left[a_{j}, b_{j}\right],\left(a_{j} \leq b_{j}, j=1,2, \cdots\right)$ to their algebraic interval numbers, which are expressed as Equation 3.1, then the product of Equation 3.1.1 and Equation 3.1.2 is defined as Equation 3.5.

$$
\begin{aligned}
A_{i} \cdot A_{j} & =\left[\min \left(A_{i} \cdot A_{j}\right), \max \left(A_{i} \cdot A_{j}\right)\right] \\
& =\left[\begin{array}{c}
\min \left\{\left(a_{i} \cdot a_{j}\right),\left(a_{i} \cdot b_{j}\right),\left(b_{i} \cdot b_{j}\right),\left(b_{i} \cdot a_{j}\right)\right\} \\
, \max \left\{\left(a_{i} \cdot a_{j}\right),\left(a_{i} \cdot b_{j}\right),\left(b_{i} \cdot b_{j}\right),\left(b_{i} \cdot a_{j}\right)\right\}
\end{array}\right]
\end{aligned}
$$

Known from Equation 3.4, the proposition is right.

Theorem $\quad 3.4 \quad$ Given interval numbers $A_{i}=\left[a_{i}, b_{i}\right],\left(a_{i} \leq b_{i}, i=1,2, \cdots\right) \quad, \quad A_{j}=\left[a_{j}, b_{j}\right]$, $\left(a_{j} \leq b_{j}\right.$, and $\left.a_{j} \neq 0, b_{j} \neq 0, j=1,2, \cdots\right)$, then the quotient of $A_{i}$ divided by $A_{j}$ is

$$
A_{i} / A_{j}=\left[\begin{array}{c}
\min \left\{\left(a_{i} / a_{j}\right),\left(a_{i} / b_{j}\right),\left(b_{i} / a_{j}\right),\left(b_{i} / b_{j}\right)\right\} \\
, \max \left\{\left(a_{i} / a_{j}\right),\left(a_{i} / b_{j}\right),\left(b_{i} / a_{j}\right),\left(b_{i} / b_{j}\right)\right\}
\end{array}\right]
$$

when algebraic interval numbers are employed as intermediate representations in the operation.

\section{CASE STUDY}

According to the characterization and operation rules of algebraic interval numbers, we take a $2 \times 2$ zero-sum interval matrix game as an example, and compute the solution of its mixed strategy as showed in Example 3.

Example 3. The problem is based on interval matrix game $G(\otimes)=\left\{S_{1}^{\otimes}, S_{2}^{\otimes}, A(\otimes)\right\}$, where $G(\otimes)$ means it is a problem of matrix game based on interval numbers, and $S_{1}^{\otimes}, S_{2}^{\otimes}$ represent player 1's and player 2's matrix game strategy based on interval numbers, $A(\otimes)$ represents the profit matrix of the game. Given $A(\otimes)$ as shown in Equation 4.1 , our task is to find the optimal solution of the game, 
compare and analyze the amplification distortion of the classic interval arithmetic and the size of the interval numbers of the optimal strategy solution.

$A(\otimes)=\left(\begin{array}{cc}{[0,1]} & {[2,3]} \\ 4 & 2\end{array}\right)=\left(\begin{array}{cc}\gamma_{11} & 2+\gamma_{12} \\ 4 & 2\end{array}\right),\left(\gamma_{11} \in[0,1], \gamma_{12} \in[0,1]\right)$

\section{Solution :}

Using the formula for $2 \times 2$ zero-sum matrix game's mixed strategy and the arithmetic operations of algebraic interval numbers provided in this paper, we have $a_{11}=\gamma_{11}, a_{12}=2+\gamma_{12}, a_{21}=4, a_{22}=2$. Player 1 and 2 's optimal mixed strategy and interval game values are respectively showed in Equation 4.2, 4.3, 4.4, 4.5 and 4.6.

$$
\begin{aligned}
x_{1}^{*}= & \frac{a_{22}-a_{21}}{\left(a_{11}+a_{22}\right)-\left(a_{12}+a_{21}\right)}=\frac{2-4}{\left(\gamma_{11}+2\right)-\left(2+\gamma_{12}+4\right)}=\frac{-2}{\gamma_{11}-\gamma_{12}-4} \\
& =\left\{\begin{array}{ll}
\frac{2}{3}, & \text { if }, \gamma_{11}=1, \gamma_{12}=0 \\
\frac{2}{5}, & \text { if }, \gamma_{11}=0, \gamma_{12}=1
\end{array}=\left[\frac{2}{5}, \frac{2}{3}\right]=\frac{2}{5}+\frac{4}{15} \gamma_{1 . x}, \gamma_{1 . x} \in[0,1]\right.
\end{aligned}
$$

$$
\begin{aligned}
x_{2}^{*}= & \frac{a_{11}-a_{12}}{\left(a_{11}+a_{22}\right)-\left(a_{12}+a_{21}\right)}=\frac{\gamma_{11}-\left(2+\gamma_{12}\right)}{\left(\gamma_{11}+2\right)-\left(2+\gamma_{12}+4\right)}=\frac{\gamma_{11}-2-\gamma_{12}}{\gamma_{11}-\gamma_{12}-4} \\
& =\left\{\begin{array}{ll}
\frac{1}{3}, & \text { if }, \gamma_{11}=1, \gamma_{12}=0 \\
\frac{3}{5}, & \text { if }, \gamma_{11}=0, \gamma_{12}=1
\end{array}=\left[\frac{1}{3}, \frac{3}{5}\right]=\frac{1}{3}+\frac{4}{15} \gamma_{2 . x}, \gamma_{2 . x} \in[0,1]\right.
\end{aligned}
$$

$$
y_{1}^{*}=\frac{a_{22}-a_{12}}{\left(a_{11}+a_{22}\right)-\left(a_{12}+a_{21}\right)}=\frac{-\gamma_{12}}{\gamma_{11}-\gamma_{12}-4}
$$$$
=\left\{\begin{array}{c}
0, \quad \text { if }, \gamma_{12}=0 \\
\frac{1}{4}, \quad \text { if }, \gamma_{11}=1, \gamma_{12}=1
\end{array}=\left[0, \frac{1}{4}\right]=\frac{1}{4} \gamma_{1 . y}, \gamma_{1 . y} \in[0,1]\right.
$$

$$
\begin{aligned}
& y_{2}^{*}=\frac{a_{11}-a_{21}}{\left(a_{11}+a_{22}\right)-\left(a_{12}+a_{21}\right)}=\frac{\gamma_{11}-4}{\gamma_{11}-\gamma_{12}-4} \\
& =\left\{\begin{array}{c}
1, \text { if } \gamma_{12}=0 \\
\frac{4}{5}, \text { if } \gamma_{11}=0, \gamma_{12}=1
\end{array}=\left[\frac{4}{5}, 1\right]=\frac{4}{5}+\frac{1}{5} \gamma_{2 . y}, \gamma_{2 . y} \in[0,1]\right.
\end{aligned}
$$

$$
\begin{aligned}
& v_{G}^{*}=\frac{a_{11} \cdot a_{22}-a_{12} \cdot a_{21}}{\left(a_{11}+a_{22}\right)-\left(a_{12}+a_{21}\right)}=\frac{2 \gamma_{11}-8-4 \gamma_{12}}{\gamma_{11}-\gamma_{12}-4} \\
& =\left\{\begin{array}{c}
2, \text { if } \gamma_{12}=0 \\
\frac{5}{2}, \text { if } \gamma_{11}=1, \gamma_{12}=1
\end{array}=\left[2, \frac{5}{2}\right]=2+\frac{1}{2} \gamma_{v, G}, \gamma_{v, G} \in[0,1]\right.
\end{aligned}
$$

The comparison of results in Table 1 shows, the classic

\begin{tabular}{|c|c|c|}
\hline & $\begin{array}{l}\text { Calculation } \\
\text { results of } \\
\text { classic }\end{array}$ & $\begin{array}{l}\text { Calculation results } \\
\text { of new } \\
\text { computation } \\
\text { method }\end{array}$ \\
\hline & A & $A_{2}$ \\
\hline$x_{1}^{*}$ & {$[2 / 5,2 / 3]$} & {$[2 / 5,2 / 3]$} \\
\hline$x_{2}^{*}$ & {$[1 / 5,1]$} & {$[1 / 3,3 / 5]$} \\
\hline$y_{1}^{*}$ & {$[0,1 / 3]$} & {$[0,1 / 4]$} \\
\hline$y_{2}^{*}$ & {$[3 / 5,4 / 3]$} & {$[4 / 5,1]$} \\
\hline$v_{G}^{*}$ & {$[6 / 5,4]$} & {$[2,5 / 2]$} \\
\hline
\end{tabular}
interval arithmetic has resulted in the amplification distortion of the results in many cases, but the results following arithmetic of the proposed algebraic intervals are consistent with the results of the classical mathematical optimization, and they do not have the amplification distortion from the classic interval arithmetic.

Table 1 Comparison of computation method of classic and standard interval number

\section{CONCLUSIONS}

This paper analyzed the possible serious diameter expansion and information distortion of the current interval arithmetic. Based on our detailed investigation of this phenomenon, we have identified the sources of this problem. Following the traditional interval arithmetic, the same interval number may take different values when they appear at more than one location in relevant operations. To solve this problem, this paper has defined an intermediate representation of interval number -- algebraic interval numbers. Based on our notion of algebraic interval numbers, we discussed their arithmetic operations and defined the operating rules. By representing interval numbers in their algebraic forms, this paper converts the operation of interval numbers to a class of optimization problems. In this way, we have effectively avoided different interpretations of the same interval number at different occurrences in the operation, and provided a fundamental solution to the diameter expansion and 
information distortion in interval operations. In the end, a detailed comparative analysis on the equilibrium results of an interval Matrix Game problem is provided to demonstrate the feasibility and reliability of the proposed model.

\section{ACKNOWLEDGMENT}

This research is supported by the National Science Foundation of China under Projects 10zd\&014, 90924022, 70971064 and 08AJY024. It is also supported by the International Joint Project sponsored by both the National Science Foundation of China and the Royal Society of UK..

\section{REFERENCES}

[1] Cerone, A. Axiomatisation of an interval calculus for theorem proving. Theoretical Computer Science 42,2001.

[2] Chen, C., Dong, J. S., Sun, J. A verification system for timed interval calculus. The 30th International Conference on Software Engineering (ICSE'08), 2008 ,271-280.

[3] Deng J. L. Application of grey system theory in China. Proceedings First International Symposium on Uncertainty Modeling and Analysis, USA, 1990,285-291.

[4] Fang Z G, Liu S F. Grey matrix game model based on pure strategy, Journal of Nanjing University of Aeronautics \& Astronautics. 2003

[5] Fang Z G, Liu S F. The military traffic flows distribute model research of maximum gray information entropy based on maximum concealment, Chinese Journal of Management Science; 2003.

[6] Fang Z G, Liu S F. Grey matrix game model based on pure strategy, Proceedings of the 32nd International Conference on Computers and Industrial Engineering, August 11-August 13, Limerick, Ireland. 2003.
[7] Ishihuchi H, Tanaka M. Multi-objective programming in optimization of the interval objective function. European Journal of Operation Research, 1990,48: 219-225.

[8] Kum C. Operations research. Operations Research, Beijing : Tsinghua University Press. 1990.

[9] Kundu S. Min-transitivity of fuzzy leftness relationship and its application to decision making. Fuzzy Sets and Systems, 1997,86: 357 367.

[10] Moore R. E. Method and application of interval analysis. London: Prentice-Hall. 1979.

[11] Moore R. E., Lodwick W. Interval analysis and fuzzy set theory. Fuzzy Sets and Systems, 2003,135:5-9.

[12] Nakahara Y, Sasaki M, Gen M. On the linear programming problems with interval coefficients. International Journal of Computer Industrial Engineering, 1992,23: 301-304.

[13] Redrycz, Park The design of granular classifiers: A study in the synergy of interval calculus and fuzzy sets in pattern recognition. 2008.

[14] Senguta A, Pal T K. On comparing interval numbers. European Journal of Operation Research, 2000 ,127: 28-43.

[15] Xu Z S, Da Q L. Research on method for ranking interval numbers. Systems Engineering, 2001, 19(6):94-96.

[16] Young R. C. The algebra of many-valued quantities. Annals of Mathematics, 1931,31: 260-290.

[17] Zhang J J. Research on method for ranking interval numbers. Operations Research and Management Science, 2003,12(3):18-22.

[18] Zhang, S. C., Zhang, C. Q. (1999) IMC: A method for interval calculus in matrix. Knowledge and Information Systems, 1(2): 257-268.

[19] Zhang, X. F., Zhang X. W. The ranking of interval numbers and its application to decision of systems. Systems Engineering - Theory \& Practice, 1999,19(7):112-115. 